\title{
NUMERICAL ANALYSIS OF A MECHANICAL DE-ICING PROCESS BY LOW FREQUENCY OSCILLATION OF A CFRP LAYER
}

\author{
F. GRUBERT ${ }^{1 *}$, M. NUÑO* AND K.-U. SCHRÖDER* \\ *Institute of Structural Mechanics and Lightweight Design \\ RWTH Aachen University \\ Wüllnerstr. 7, 52064 Aachen, Germany \\ www.sla.rwth-aachen.de \\ ${ }^{1}$ felix.grubert@sla.rwth-aachen.de
}

Key words: General Aviation, Ice, Mechanical De-Icing, Oscillation, CFRP.

\begin{abstract}
De-icing of general aviation aircraft is usually realized by chemical or thermal processes. These lead to an increase in fuel consumption or usage of electrical energy. Mechanical de-icing is a way to significantly reduce this consumption. In mechanical de-icing, a surface is deformed so that the required failure mechanisms are induced in the ice, causing it to detach.

In this paper, the release behavior of ice on a CFRP layer is investigated. The CFRP layer consists of two plies, each $0.3 \mathrm{~mm}$ thick. A numerical calculation is performed to determine the natural frequency and the required amplitudes of the vibration. In addition, the relationship between the various failure mechanisms of ice and the ice layer thickness, as well as the control values are determined. Tests are also being conducted to demonstrate the feasibility of mechanical de-icing. For this purpose, the surface of a CFRP layer is iced with water in a climate chamber at $-20^{\circ} \mathrm{C}$. A modal shaker is connected to the CFRP and generates the required displacements at desired frequencies to observe the detachment of the ice.
\end{abstract}

\section{INTRODUCTION}

De-icing of aircraft is usually realized by chemical or thermal processes. These processes became well established in the past decades, although they have some disadvantages. The increased consumption of fuel or the additional amount of electrical energy have always been accepted by CS-25 aircraft due to their relatively short flight time in icing conditions. For the increasing regional air traffic in the future, where vehicles will be in icing conditions for longer periods and will also increasingly use electric propulsion systems, efficient design of all systems 
is of highest importance. To make the de-icing system more efficient, an electro-mechanical approach can be adopted.

In this paper, the basic feasibility of mechanical de-icing is demonstrated. At the beginning, established de-icing systems are presented and parameters are calculated that allow a quantitative classification. The calculations always refer to the geometry of the reference aircraft, the Silent Air Taxi (SAT). The most important geometric parameters are listed in Table 1. The calculations also refer to an ambient temperature of $15^{\circ} \mathrm{C}$ on the ground and a flight in continuous maximum icing conditions. After that, different failure mechanisms of ice are presented and the influence of the surface material is discussed. In section 4, de-icing tests are performed. For this purpose, modal analyses are carried out to determine resonance frequencies and after that, the feasibility of mechanical de-icing is demonstrated. Finally, numerical models are created to represent the knowledge gained from the experiments and to show a correlation between failure mechanisms and de-icing time.
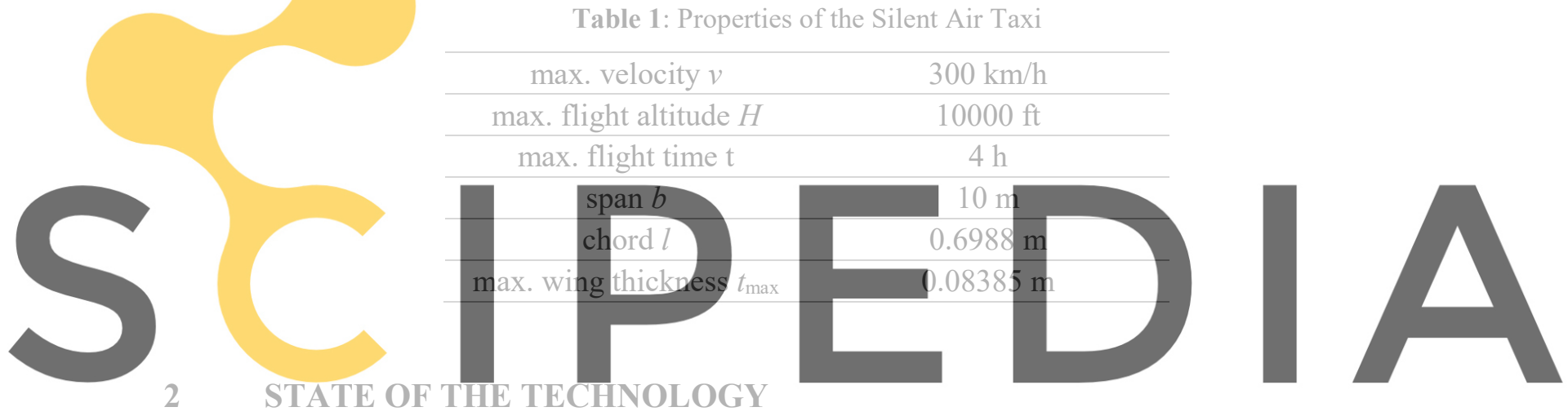

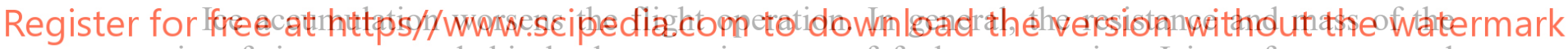
aircraft increases and this leads to an increase of fuel consumption. Icing of sensors and antennas can lead to incorrectly transmitted data or hinder communication. In addition, ice at the lifting surfaces leads to a reduction of the lift coefficient and therefore changes the flight characteristics.[1]

In order to prevent the danger of iced lifting surfaces, three different systems are used today. One of these systems is fluid based. It works by pumping a glycol-containing liquid onto the wing surface, where it mixes with the impinging water and lowers the freezing point. With a lower freezing point the water does not freeze at ambient temperature at the respective flight level. Kohlmann et al. [2] have established a calculation method that can be used to determine the amount of de-icing fluid. The mass $M_{\mathrm{F}}$ of the de-icing fluid is calculated with

$$
M_{F}=\frac{G M_{W} t b}{X-G}
$$

$G$ is the glycol mass fraction in the fluid-water-mixture and equals $12 \%, X$ is the glycol mass fraction in the de-icing fluid and equals $80 \%$. $G$ depends on the temperature above which the impinging water is not allowed to freeze, which is the ambient temperature. $M_{\mathrm{W}}$ is the water 
catchment and is calculated in (6). The mass of de-icing fluid needed for a flight of the SAT in continuous maximum icing conditions is thus $20.97 \mathrm{~kg}$.

Another system is the electro-thermal system. This system involves the installation of heating mats in the leading wing edge, which can melt ice or evaporate water. This system has a low mass but the big disadvantage of a very high demand of electrical energy.[1] The calculation of the required heat flow is based on methods of the Aerospace Information Report AIR1168/4.[3] The heat flow is composed of three parts, convection, heating and evaporation:

$$
\begin{gathered}
q=q_{\text {conv }}+q_{\text {heat }}+q_{\text {evap }} \\
q_{\text {conv }}=\alpha\left[T_{\text {surf }}-\left(T_{\text {amb }}+\Delta T\right)\right] \\
q_{\text {heat }}=M_{W} c_{W}\left[T_{\text {surf }}-\left(T_{\text {amb }}+\Delta T\right)\right] \\
q_{\text {evap }}=M_{W} \Delta h_{v}
\end{gathered}
$$

The heat transfer coefficient $\alpha$ is determined using different key ratios with the assumption of a longitudinally flowed plate. The surface temperature $T_{\text {surf }}$ can be determined graphically. The boundary layer heating $\Delta T$ is quadratic to the velocity. $c \mathrm{~W}$ is the heat capacity and $\Delta h_{\mathrm{v}}$ the evaporation enthalpy of water. The water catchment is calculated with the water catch

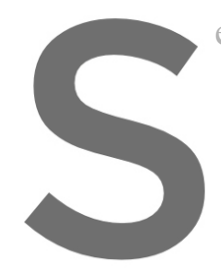
efficiency $E$ and the liquid water conten $L W C$ as follows:
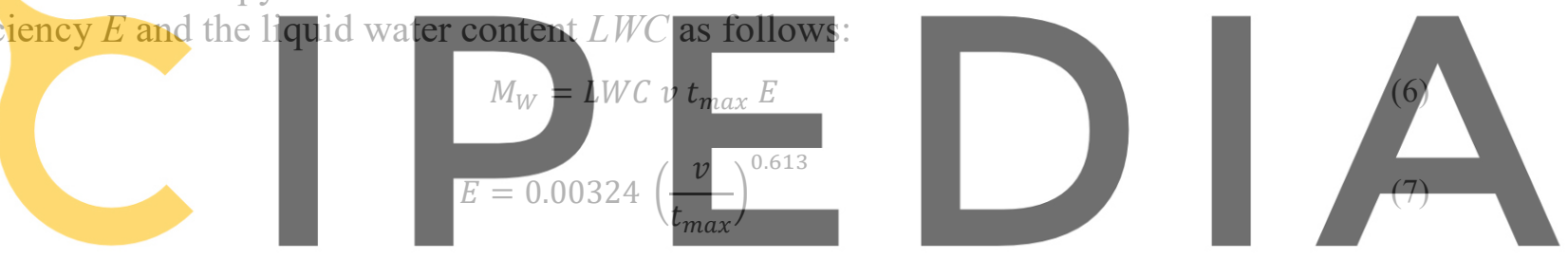

Thus, the heat flow which must act on the impinging water amounts to $35.8 \mathrm{~kW}$ according

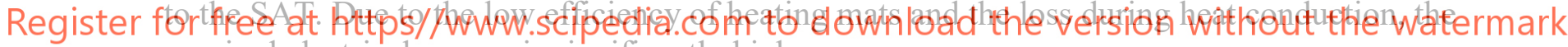
required electrical energy is significantly higher.

One system that also uses thermal energy is the bleed air system. Here, bleed air is extracted

from the engine and guided along the leading edge of the wing. However, this system only works on engines where combustion takes place and warm bleed air can be extracted. For this reason, this de-icing method is not available for all-electric engines.

CS-25 aircraft fly through icing conditions only for short periods of time due to their high flight level and accept the major disadvantages of established de-icing systems. General aviation aircraft, such as future air taxes, may be exposed to icing conditions for the entire flight time. Therefore, their de-icing system must be designed much more efficiently. One solution is to use a mechanical de-icing system. 


\section{ICE BEHAVIOR}

Mechanical de-icing systems generate de-icing by deforming and bending the surface so that different failure mechanisms are triggered in the ice.[1] In this work, two failure mechanisms will be focused. Overmeyer et al. [4] have described that ice is detached when a certain shear stress is reached in the contact area between ice and wing surface. The magnitude of the shear stress depends on the properties of the used surface material and the adhesion forces between ice and surface. Hejazi et al. [5] and Strobl et al. [6] have experimentally determined the required shear stresses for different surface material. However, since there are no uniform testing procedures yet, there are large deviations. Table 2 lists some values of shear stresses in relation to different materials.

Table 2: Required shear stress for ice detachment for different materials $[5,6]$

\begin{tabular}{cc} 
Material & Shear stress [kPa] \\
\hline Brass & 252.1 \\
\hline Stainless steel & 173.67 \\
\hline GFRP & 94.73 \\
\hline Polyurethane & 25 \\
\hline
\end{tabular}

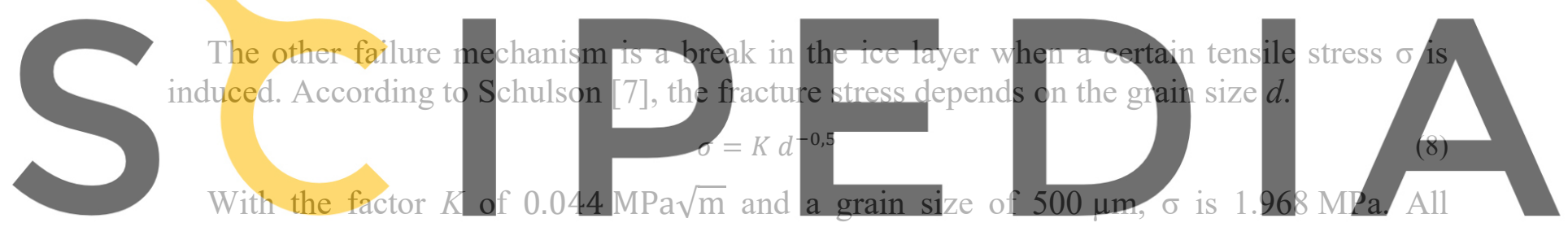

properties of ice assumed in this work are listed in Table 3:

Register for free at https//www.scipedia.com to download the version without the watermark

\begin{tabular}{lc}
\hline Density $\rho$ & $916,7 \mathrm{~kg} / \mathrm{m}^{3}$ \\
\hline Young's modulus E & $10 \mathrm{GPa}$ \\
\hline Poisson's ratio $v$ & 0,33 \\
\hline Fracture tensile stress $\sigma$ & $1,968 \mathrm{MPa}$ \\
\hline
\end{tabular}

To trigger these failure mechanisms, the surface with the ice layer must be bent. This bending can be applied by large, one-time deformation, such as in the use of de-icing boots, or due to an oscillating deformation. However, a lot of energy is required for large, one-time deformation, as large forces are required.[1] By exploiting vibrations with excitation frequencies close to the resonant frequency, a sufficiently large deformation can be achieved even with small forces.[9] This principle is investigated in this work. 


\section{EXPERIMENT}

Experiments were conducted to demonstrate the feasibility of mechanical de-icing by oscillations and to determine a relationship between required force and failure behavior of the ice. First, modal analyses were performed to determine the resonant frequencies of the system, and afterwards de-icing tests were carried out.

\subsection{Test set-up}

Since these tests are intended to be feasibility tests, they were performed on a simple plate demonstrator and not on the geometry of a wing leading edge. This plate demonstrator consists of a CFRP plate (2 CFRP plies: $0,3 \mathrm{~mm}$ thick each, $+/-45^{\circ}$ fiber orientation) and a layer of ice on top of it. To freeze the plate, it was placed in a climate chamber at $-20^{\circ} \mathrm{C}$ and water was poured over it. This does not match the icing conditions in flight, where supercooled water droplets hit the wing surface and freeze, but it could not be realized any other way due to the given infrastructure. After freezing, the plate demonstrator was fully clamped in a frame structure on two sides and fixed with a modal shaker using a bonded screw. A force and acceleration sensor records data during the experiments to detect resonance frequencies. Figure 1 shows the test set-up.
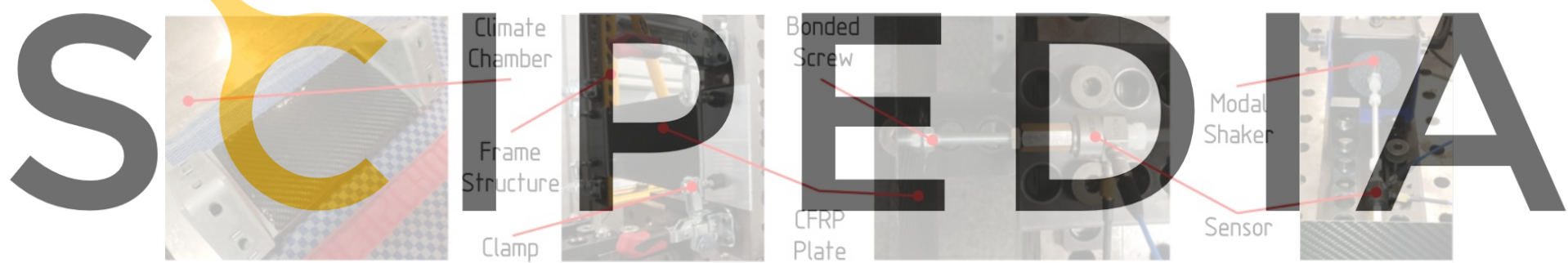

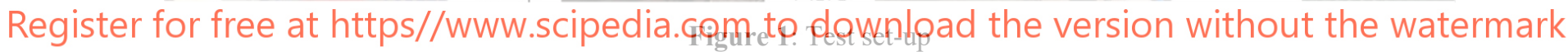

\subsection{Modal analysis}

Modal Analysis were performed to determine the resonant frequencies. During the analysis, the frequencies from 1 to $600 \mathrm{~Hz}$ were approached within 180 seconds and acceleration and force were recorded. Figure 2 shows the results of the modal analysis of the plate demonstrator without ice. Resonance is detected at the peaks, at $32 \mathrm{~Hz}, 109 \mathrm{~Hz}, 151 \mathrm{~Hz}, 179 \mathrm{~Hz}$ and $457 \mathrm{~Hz}$. 


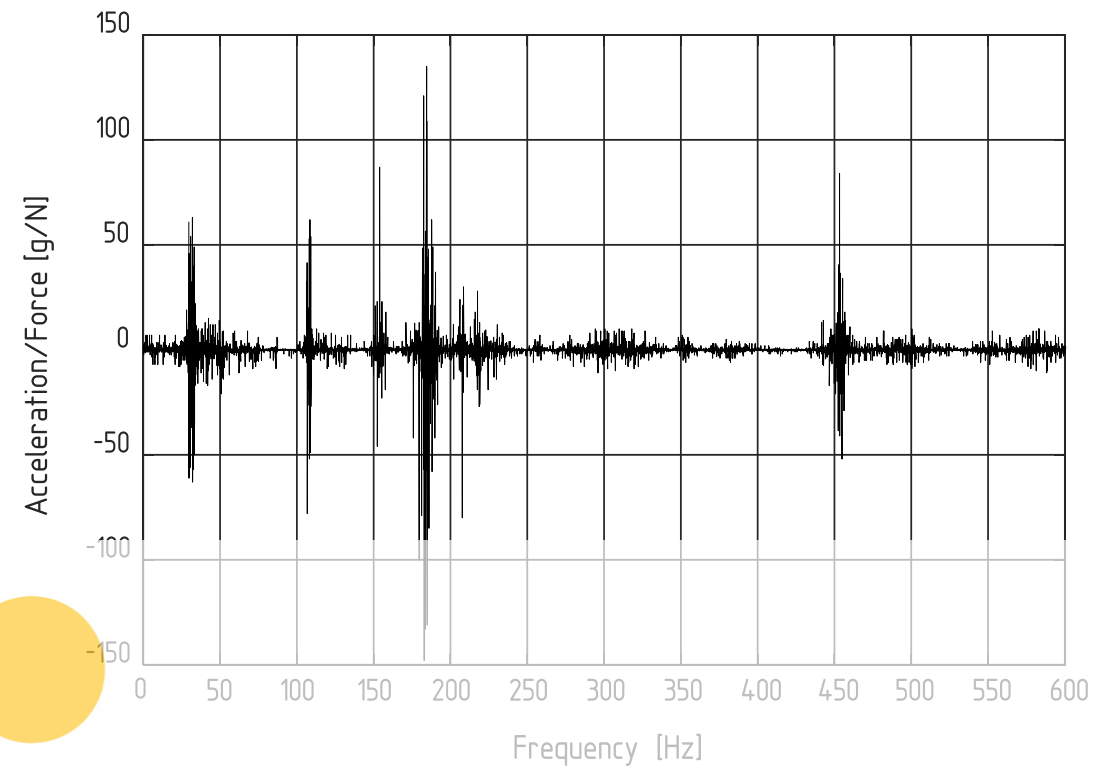

Figure 2: Modal analysis of CFRP plate without ice

Figure 3 shows the results of the modal analysis of the plate demonstrator with ice. This time, acceleration and force are plotted over frequency. All tests showed reso $79 \pm 3.95 \mathrm{~Hz}$, where acceleration became maximum und force amplitude minimun.
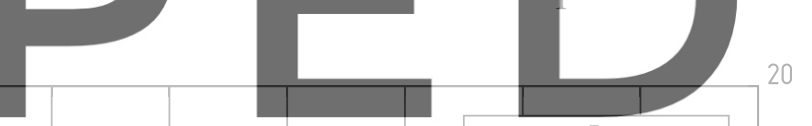
20
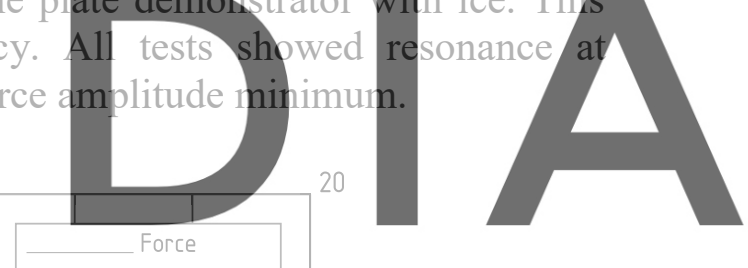

Register for free at http\$//www.scipedia.com to download the version without the watermark

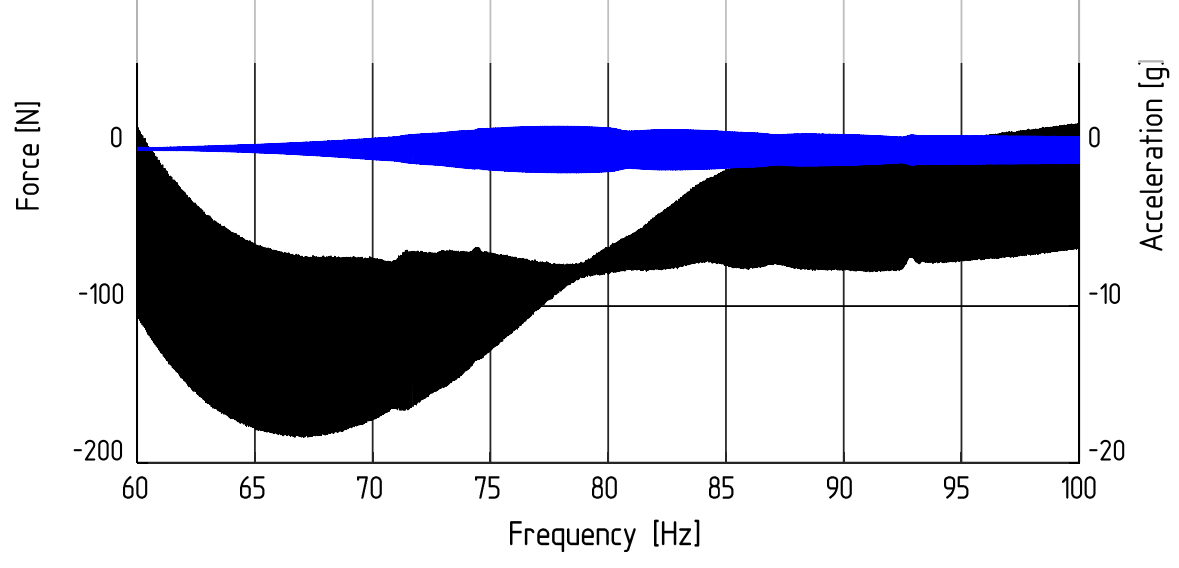

Figure 3: Modal analysis of CFRP plate with ice 


\subsection{De-icing tests}

Subsequently, de-icing tests were carried out. For this purpose, the test specimen was excited at the previously determined resonant frequency and only the input voltage of the modal shaker and thus the deformation were varied. De-icing can only be observed optically, as there are no external forces acting like an air flow which could detach the ice. Figure 4 shows the results of one de-icing test.
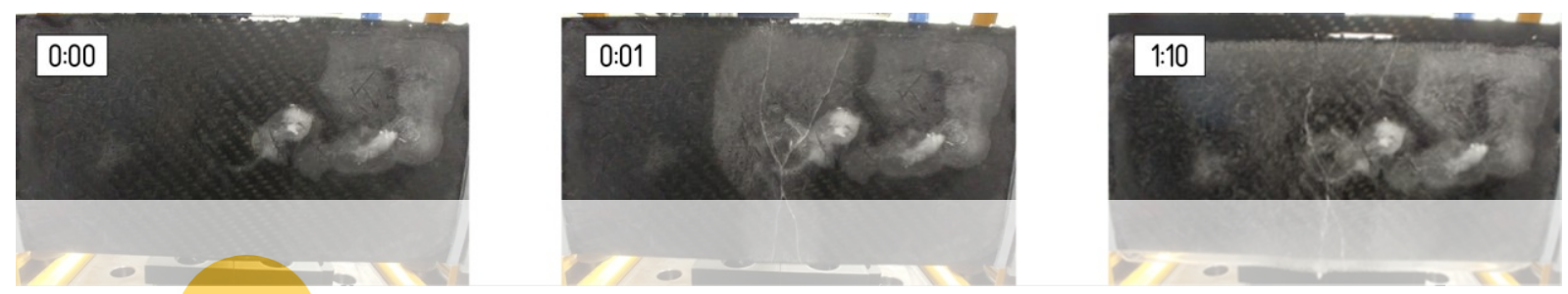

Figure 4: Photos of iced CFRP during de-icing test

At 0:00 a nearly homogeneous ice layer is visible. The impurities in the ice layer are caused by uneven freezing. After less than one second, a fracture appears in the center of the test specimen and the ice detaches from the CFRP surface in the area immediately around the fracture (area changes color). Since the tests could not be performed inside the climatic chamber, the ice melts after about three minutes, which is why the ice layer slips of sheet even without vibration. However, after de-ieing this clear sign of ice detachment and thus of successful de-icing. In a occurred, no forces could be recorded because the the basic objective of this work, to show a first feasibility of no In all at this point, even if no direct correlation between de-icing time and vibration amplitude could

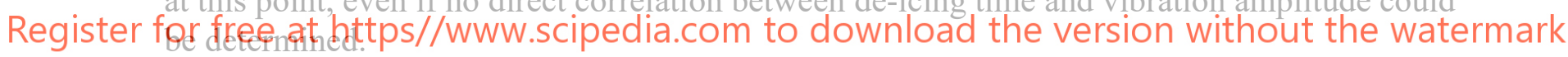




\section{NUMERICS}

In the numerics, the knowledge gained from the experiments is reproduced and an adaptation to other geometries, such as different ice layer thicknesses, is carried out. For this purpose, modal analyses are performed first and then the relationship between excitation force and deicing time is determined for different ice layer thicknesses.

For pre- and post-processing Hyperworks is used. The simulations are calculated with the solver Abaqus. The CFRP plate and the ice layer are modeled with shell elements and connected as a tie. The CFRP is fully clamped on the two short sides and excited in the middle by an oscillating force, analogous to the experiment.

\subsection{Modal analysis}

In Figure 5, the resonance frequency is plotted over different ice layer thicknesses. The frequency of $32 \mathrm{~Hz}$, which was determined for the CFRP plate without ice in the experiments, is also achieved by numerical simulations. The validation of the numerical model with regard to the CFRP plate was thus successful.

In the experiments, an ice thickness of $5 \mathrm{~mm}$ was always produced. The resonance frequency measured $(79 \mathrm{~Hz})$ could not be reproduced in the numerics. This is because the exact properties of the ice created in the experiments are unknown and values from literature were assumed. It

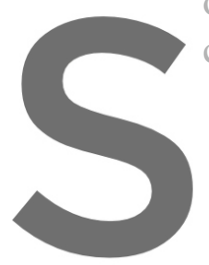

can be assumed that the
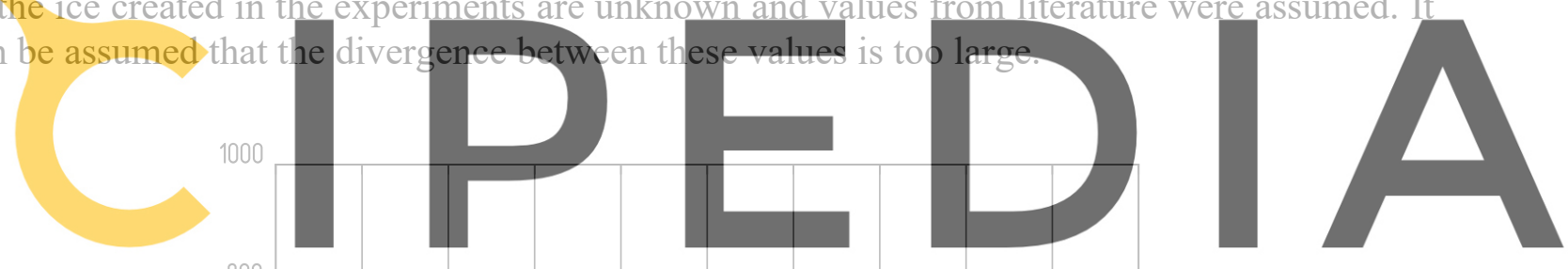

Register for free at https//www.scipedia.com to download the yersion without the watermark

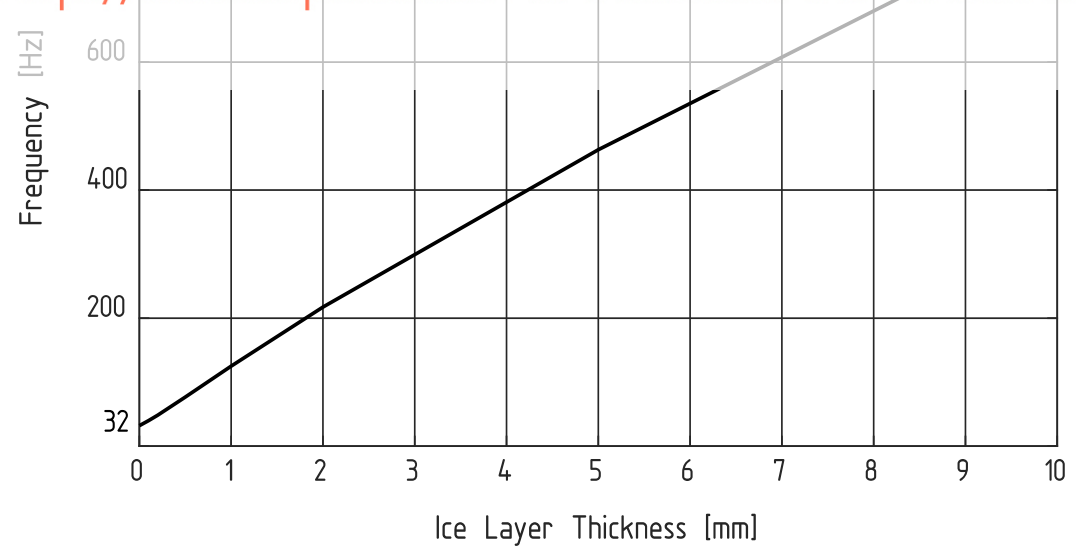

Figure 5: Resonant frequency plotted over ice layer thickness 


\subsection{De-icing modeling}

In addition, an attempt was made to model the de-icing process. For this purpose, a correlation between de-icing time and oscillation force was determined. It was always assumed that an intact and homogeneous ice layer is oscillated up to the respective breaking and detachment stresses. The value in contact with GFRP is chosen as the detachment stress, since CFRP and GFRP have similar surface properties and the literature does not provide a value specifically determined for CFRP. Figure 6 shows the results:

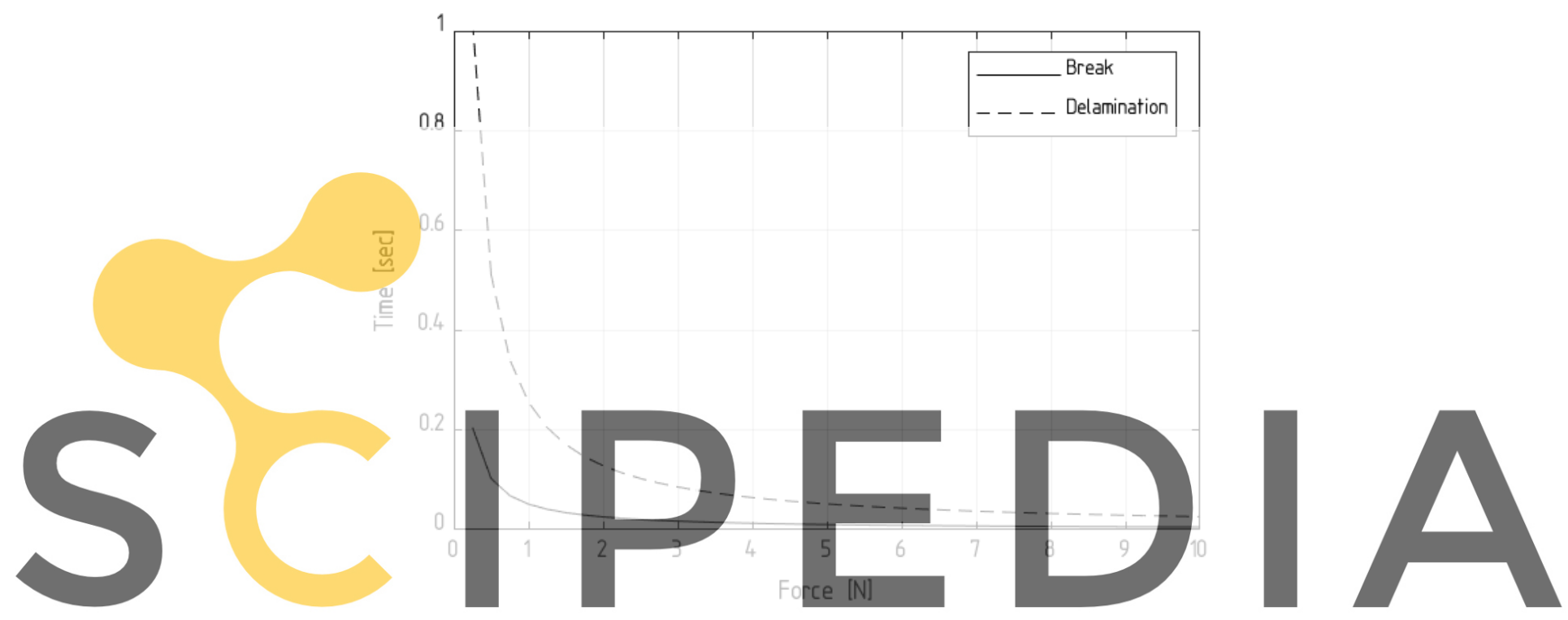

Figure 6: Break- and delamination time plotted over force at $5 \mathrm{~mm}$ ice layer thickness
Register for free at https//WWW.scipedia.com to download the version without the watermark

The greater the force, the shorter the time to fracture and detachment of the ice. At this point, however, it is difficult to compare the results with those of the experiments. In both, the time to fracture and detachment in the middle area of the ice is less than one second. However, since the ice properties of numerics and experiments are different, a direct comparison of the results is not possible. In order to allow comparability in the future, exact properties of the ice must be determined in the experiments in order to use these values in numerical simulations.

\section{CONCLUSION AND OUTLOOK}

In this paper, the basic feasibility of mechanical de-icing was demonstrated. For this purpose, established de-icing systems were presented at the beginning and characteristic values were calculated. The fluid-based system requires a large mass of de-icing fluid to be carried during the flight. For electro-thermal de-icing, a large amount of energy is required. A system that requires less energy is the electro-mechanical de-icing system. To understand the effects of mechanical de-icing, two failure mechanisms of ice were presented. Experiments were conducted to prove the feasibility. They have shown that mechanical de-icing works. 
Nevertheless, there is much potential for improvement. First, the mechanical properties of the ice have to be determined. This primarily leads to a higher accuracy of the numerical models. In addition, it is then possible to estimate how the ice generated in the climate chamber approximates the real ice as it occurs in icing conditions in flight. To obtain an even higher replication of the real ice, the experiments should be conducted in an ice wind tunnel. This would allow a more accurate ice-surface interaction as well as provide a better optical measurement due to the aerodynamic forces. In the end, another excitation concept for the oscillation must be selected, since a modal shaker would certainly be too heavy for use in aircraft.

\section{REFERENCES}

[1] Thomas, S. K., Cassoni, R. P. and MacArthur, C. D. Aircraft Anti-Icing and De-Icing Techniques and Modeling, Journal of Aircraft, Vol. 33, No. 5 (1996).

[2] Kohlmann, D. L. and Albright, A. E. A Method of Predicting Flow Rates Required to Achieve Anti-Icing Performance with a Porous Leading Edge Ice Protection System, NASA Contractor Report, No. 168213 (2013).

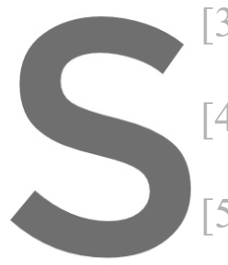

3] SAE International. Protection, Aerospace Information

4] Overmeyer, A., Palac Ice Testing, AIAA

5] Hejazi, V., Sobolev, K. and Nosonovsky, forces and interaction andtysis, Scientific
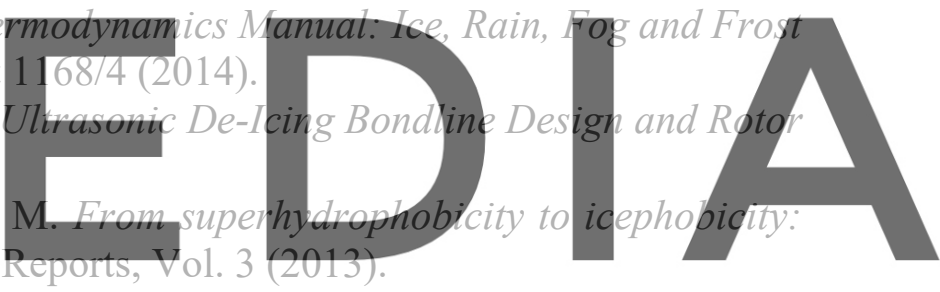

[6] Strobl, T., Raps, D. and Hornung, M. Comparative Evaluation of Ice Adhesion Behavior,

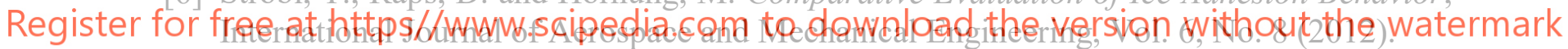

[7] Schulson, E. M. Brittle Failure of Ice, Engineering Fracture Mechanics, Vol. 68 (2001).

[8] Petrovic, J. J. Mechanical properties of ice and snow, Jounal of Materials Science, Vol. 38 (2003).

[9] Endres, M. et al. Experimental study of two electro-mechanical de-icing systems applied on a wing section tested in an icing wind tunnel, CEAS Aeronaut Journal, Vol. 8 (2017). 